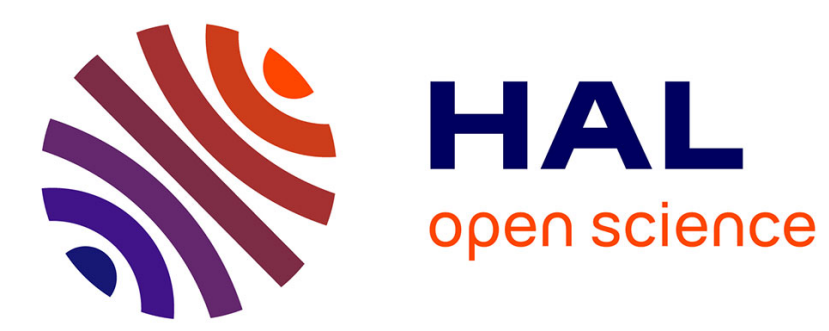

\title{
CONTAMINATION DU LAIT PAR LES RADIOÉLÉMENTS ARTIFICIELS
}

\author{
J. Morre
}

\section{To cite this version:}

J. Morre. CONTAMINATION DU LAIT PAR LES RADIOÉLÉMENTS ARTIFICIELS. Le Lait, 1968, 48 (478), pp.536-546. hal-00928472

\section{HAL Id: hal-00928472 \\ https://hal.science/hal-00928472}

Submitted on 1 Jan 1968

HAL is a multi-disciplinary open access archive for the deposit and dissemination of scientific research documents, whether they are published or not. The documents may come from teaching and research institutions in France or abroad, or from public or private research centers.
L'archive ouverte pluridisciplinaire HAL, est destinée au dépôt et à la diffusion de documents scientifiques de niveau recherche, publiés ou non, émanant des établissements d'enseignement et de recherche français ou étrangers, des laboratoires publics ou privés. 


\title{
CONTAMINATION DU LAIT PAR LES RADIOÉLÉMENTS ARTIFICIELS (1)
}

\author{
par \\ J. MORRE \\ Chef du laboratoire de radiobiologie \\ du Service vétérinaire de la Préfecture de Police
}

Aux contaminations déjà trop nombreuses qui viennent souiller le lait, s'ajoutent depuis quelques années, de nouveaux éléments dus au développement des sciences nucléaires : il s'agit des radionuclides.

Pondéralement, ces composés, même s'ils sont doués d'un grand pouvoir chimiotoxique, n'ont aucune importance, car ils n'entrent que pour une fraction extraordinairement infime dans la composition du lait, de l'ordre du dix milliardième de gramme par litre; par contre, ils émettent des rayonnements $\beta$ et $\gamma$ qui viennent, après ingestion du lait contaminé, irradier le consommateur, ce qui représente un réel danger.

\section{Origine des radiocontaminants}

Quand une bombe atomique explose dans l'atmosphère, il y a production d'une énorme quantité de produits radioactifs, une part importante tombe directement sur le polygone de tir, dans un lieu étroitement surveillé, une autre partie est entraînée au loin sous forme de particules extrêmement légères et, grâce aux vents et à la rotation de la terre, le nuage radioactif fait plusieurs fois le tour de la terre avant de se sédimenter. Il s'écoule de 3 à 5 ans avant la fin de la retombée.

Par chance, parmi les corps radioactifs créés, de très nombreux ont une période physique courte (2), si bien que par le jeu de la décroissance, l'activité du nuage diminue très rapidement. Malheureusement, certains corps, ou bien sont produits en assez grande quantité comme l'iode 131 , ou bien ont une période longue comme

(1) Bull. Soc. sc. Hyg. al., 1967, 55, nos 4-5-6, 149.

(2) Période physique ou demi-vie des Anglo-saxons, temps nécessaire pour que l'activité du radioélément ait décru de moitié. Elle varie de quelques secondes (Rh 106: 30 secondes) à des milliers d'années (C 14: 5600 ans). 
TABLEAU I

Principaux radioḱléments produits dans la fission

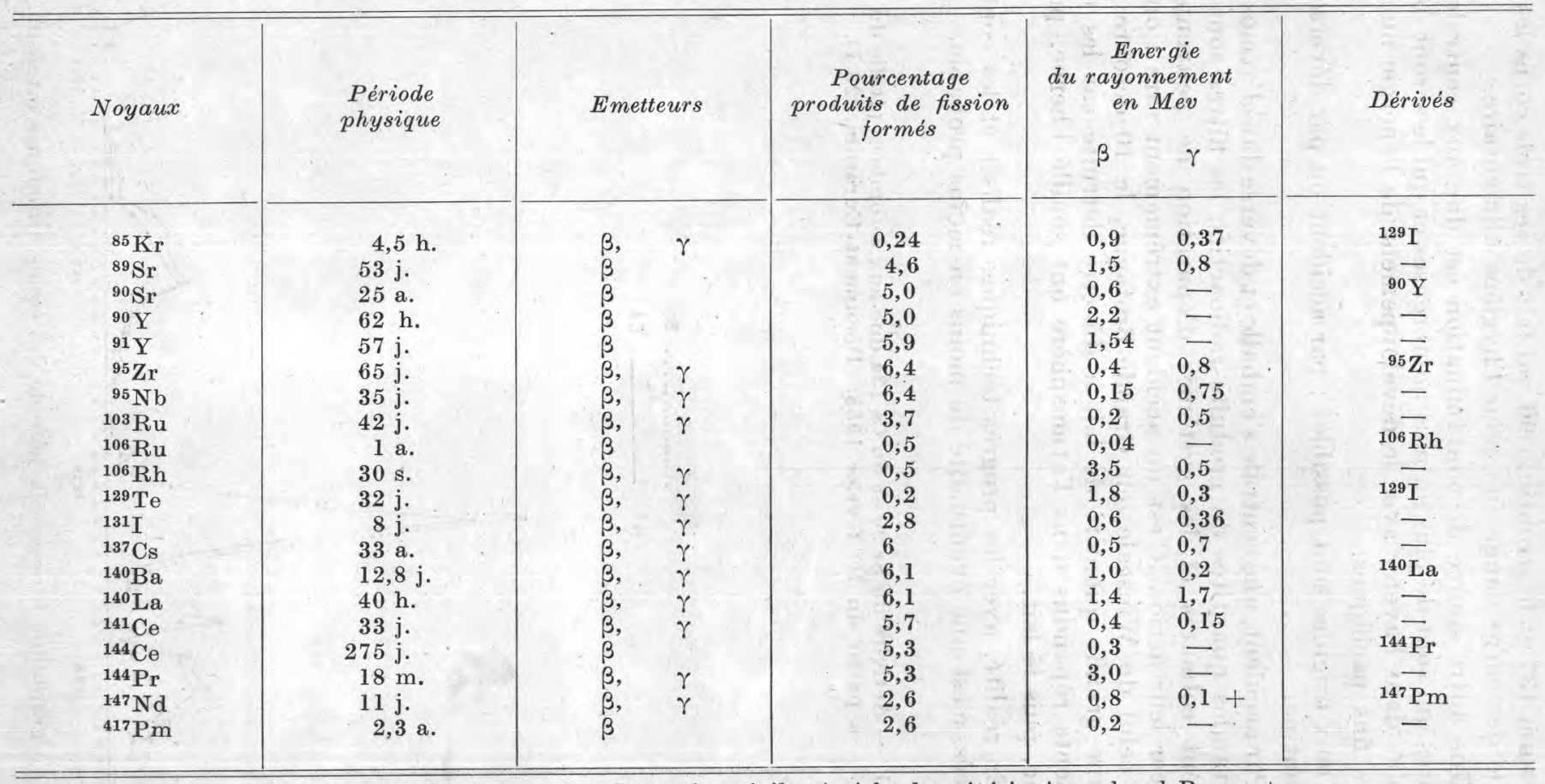

Tableau extrait de la revue Protection civile. Article du vétérinaire-colonel Boequet. 
le césium 137 et le strontium 90 qui fait de ces trois corps les ohefs de file des corps dangereux pour l'hygiène alimentaire.

Une autre source de contamination est due aux centrales nucléaires qui sont de plus en plus nombreuses et qui le seront de plus en plus dans l'avenir avec le développement de l'énergie nucléaire à des fins pacifiques.

Deux origines sont possibles : par accident ou par déversement de routine.

Par accident, une centrale s'emballe et déverse dans l'atmosphère de grandes quantités de produits radioactifs, les filtres sont rapidement colmatés et les systèmes d'épuration ne peuvent plus exercer leur action. C'est un accident extrêmement rare : on connaît celui de Winscale, en Grande-Bretagne, le 10 octobre 1957, où une grande quantité de lait a dû être détruite car les radioéléments répandus dans l'atmosphère ont souillé l'herbe, puis la vache, puis le lait.

En réalité, avec les progrès techniques réalisés et les contrôles exercés, c'est une éventualité de moins en moins probable.

Fig. 1. - Activité du Sr 90 et du Cs 134 due aux retombées (Italie du Nord) à partir du ler février 1958. (Document Euratom $223 \mathrm{I}$ ).

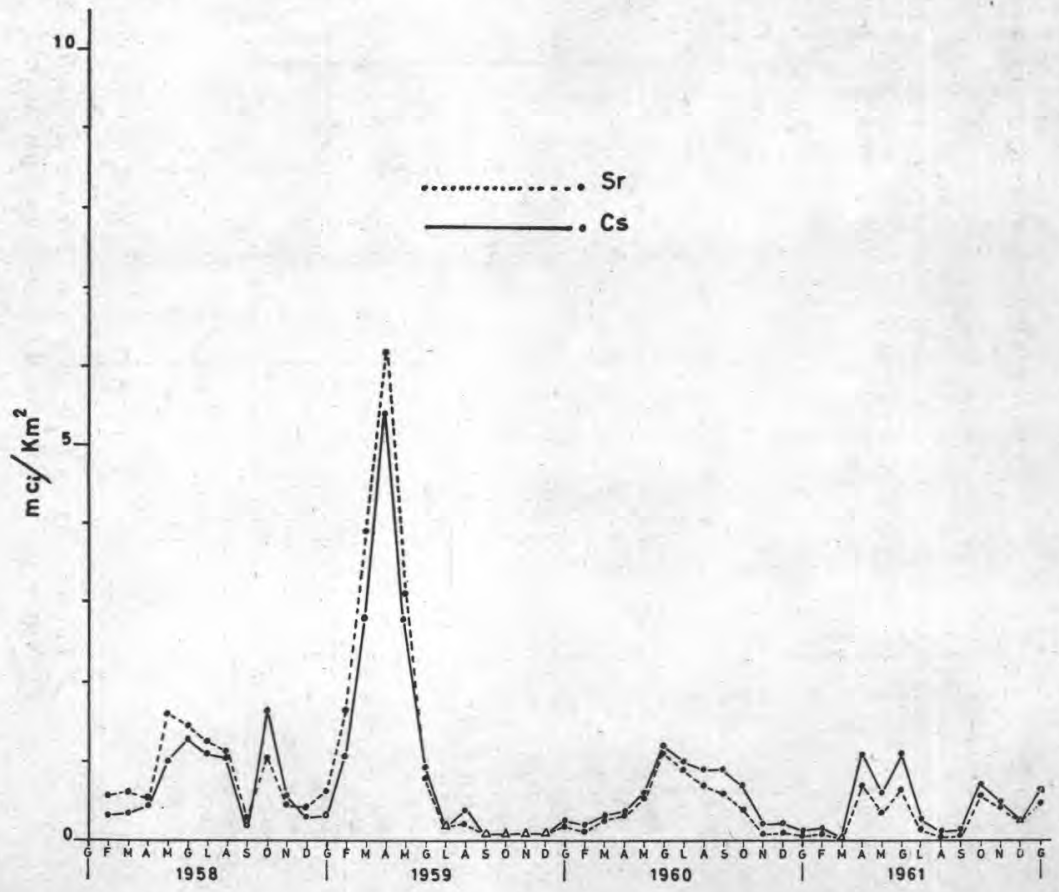

Ce graphique intéresse la période des essais atomiques intensifs. 
Par routine, le risque de pollution provient du rejet dans l'atmosphère (et éventuellement dans les eaux) de produits faiblement radioactifs mais qui peuvent, par suite d'accumulation ou de conditions météorologiques particulières, s'inclure dans la chaîne alimentaire. Là aussi, le contrôle autour des usines est sérieux et le danger faible. C'est donc, en résumé, les retombées radioactives dues aux essais nucléaires dans l'atmosphère qui représentent le plus gros danger de pollution.

\section{Mécanisme de pollution du lait}

Quelle que soit l'origine des radioéléments, la voie de pénétration est la suivante :

En suspension dans l'air, les poussières tombent peu à peu. Quand elles atteignent les couches inférieures de l'atmosphère, elles sont entraînées par la pluie vers le sol. L'absorption pulmonaire est négligeable, mais l'herbe se trouve alors souillée, puis par l'herbe le ruminant qui consomme cette herbe : une vache tond chaque jour une grande surface, done se contamine rapidement.

A côté de cette voie directe, il en existe une deuxième : le radionuclide est absorbé par le sol, repris par la plante qui est consommée. On voit immédiatement qu'il y a deux phénomènes opposés qui entrent en jeu. Il y a une dilution dans le sol avec rétention éventuelle, c'est le cas, en particulier, du césium qui est retenu facilement par les argiles. D'autre part, il y a une possibilité de concentration par la plante, ceci joue pour le strontium principalement. Pour l'iode, l'absorption est directe : pluie, herbe, vache, sans passage par le sol.

La plus grande partie des radionuclides est rejetée sans être absorbée, mais d'autres sont métabolisés : le strontium se fixe dans l'os, le césium dans le muscle et les organes génitaux, l'iode dans la thyroïde. Enfin, une partie de ces éléments est éliminée par le lait qui va à son tour contaminer l'homme.

\section{Principaux contaminants et leur métabolisme}

De tous les radionuclides produits par une explosion nucléaire, on ne retrouve dans le lait que l'iode 131 , les strontiums 90 et 89 et le césium 137 avec un peu de baryum 140 et de lanthane 140.

Le principal de tous et le plus dangereux est l'iode 131. Il a une période physique de 8 jours 08 , ce qui est peu; done, très rapidement, ce radionuclide va disparaître de l'organisme, mais il faut savoir qu'il est produit en quantités relativement importantes et, d'autre part, il se fixe électivement sur la thyroïde où il se concentre. Cette glande risque done de subir une irradiation intense. 
Sous forme d'hormone thyroïdienne, il circule dans le sang. Enfin, il est éliminé par le rein ou par les voies biliaires, mais il risque alors d'être réabsorbé par l'intestin.

On voit l'importance chez le jeune enfant chez qui la thyroïde est très active et dont le lait est la nourriture principale.

Le deuxième radiocontaminant, par ordre d'importance, est le strontium 90 dont la période physique est de 28 ans, donc très longue. Le strontium est un alcalino-terreux et suit, de ce fait, le métabolisme du calcium. L'organisme distingue mal calcium et strontium ; en cas de pénurie, le strontium est métabolisé et absorbé puis fixé dans les os. La moelle osseuse, qui est l'organe hémopoḯtique par excellence, va de ce fait, être soumise à un rayonnement $\beta$, certes de faible énergie, mais intense, car le radionuclide est situé très près de l'organe sensible.

L'enfant qui construit son squelette à partir du calcium du lait se trouve done menacé.

A côté du strontium 90 se trouve le strontium 89 qui a le même métabolisme, mais qui est beaucoup moins dangereux, car sa période physique est courte : 50 jours 40 .

Le dernier des radiocontaminants est le césium 137 dont la période physique est de 30 ans. Le césium est chimiquement très voisin du potassium, c'est un alcalin : son métabolisme est identique, aussi va-t-il se fixer dans l'intérieur de la cellule, principalement dans le muscle et dans l'appareil génital, d'où un certain danger pour les cellules nobles de l'organisme.

L'importance des autres radio-nuclides est mineure à eôté du strontium, du césium et de l'iode.

\section{Mesure de la radiocontamination du lait}

\section{Comment va-t-on mesurer cette pollution ?}

Le lait étant un produit biologique bien défini, excrété par la mamelle, ne contient qu'un nombre réduit de radio isotopes : iode 131, strontium 90 et 89 et césium 137, ce qui facilite la recherche et le dosage. D'autre part, les appareils de mesure, en particulier les spectromètres $\gamma$ ont fait d'énormes progrès ainsi que les techniques de micro-dosage chimique.

\section{lode 131}

L'iode radioactif étant un émetteur $\gamma$ peut être décelé par spectrométrie. Le seuil de détection se situe autour de 5 à $10 \mathrm{p} \mathrm{Ci} / \mathrm{1}(1)$

(1) pCi ou picocurie : $10^{-12}$ curies ; la curie correspond à l'activité d'un corps radioactif présentant $3,7 \quad 10^{-10}$ désintégrations par seconde. 
ce qui est très en dessous des normes admises en hygiène atomique. La mesure s'effectue sur le lait frais directement sans aucune préparation. La hauteur du pic permet de doser le radio-élément.

Fig. 2. - Spectre gamma établi à partir du résidu sec de six litres de lait (Italie du Nord, novembre 1961) (Document Euratom 223 I).

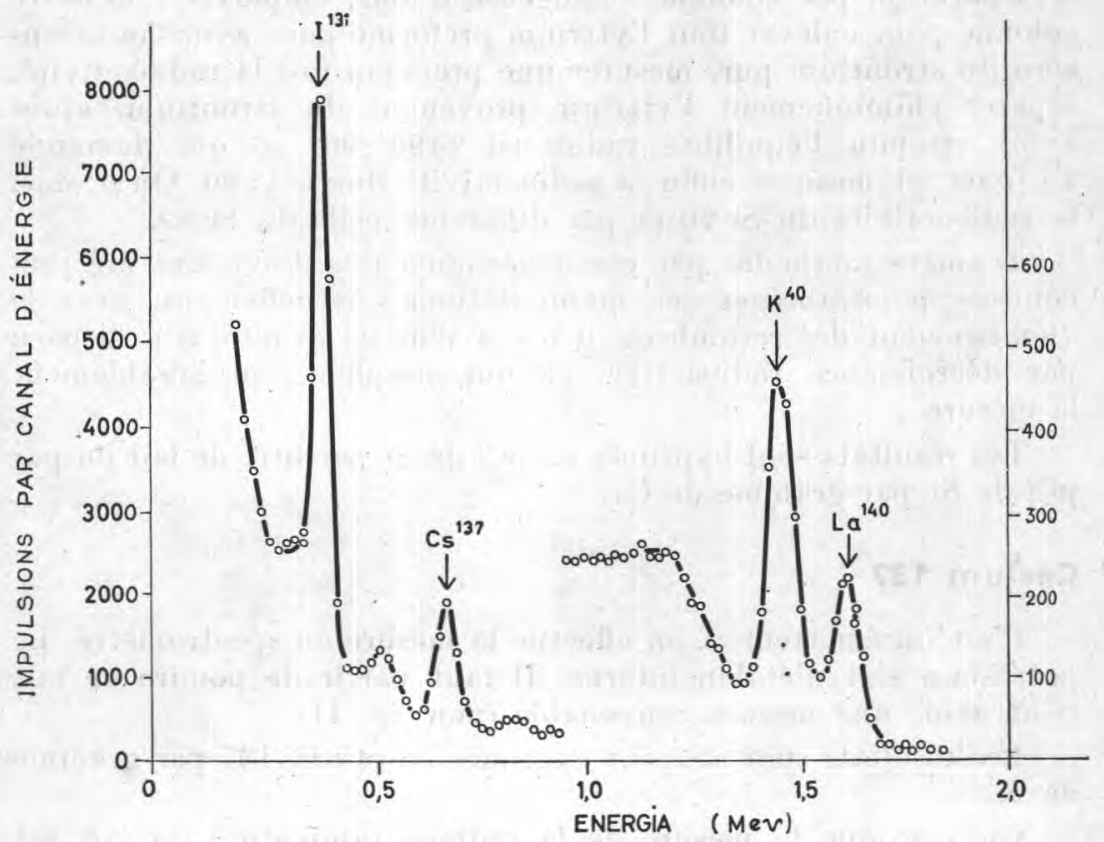

Bien noter que l'échelle des ordonnées du spectre de gauche est 10 fois celle de droite.

\section{Strontium 90 et 89}

Les deux isotopes 90 et 89 sont des émetteurs $\beta$ exclusifs et peu énergiques $(0,6 \mathrm{Mev})(1)$ donc difficiles à mesurer. D'autre part, l'isolement est rendu laborieux par la présence du calcium, qui vient troubler les réactions. La décroissance radioactive du strontium 90 produit de l'yttrium 90 (période 65 heures), il émet des $\beta$ de 2,2 Mev. Comme il appartient au groupe chimique des terres rares, il peut être assez facilement séparé du calcium et du strontium.

(1) Mev ou million d'électron-volts est une unité d'énergie : $1 \mathrm{Mev}=$ $1,59 \cdot 10^{-19}$ joules. L'énergie d'un élément radioactif permet de le caractériser sur un spectre $\gamma$. 
D'autre part, l'yttrium 89 provenant du strontium 89 est stable, ce qui permet par différence d'évaluer la radioactivité due à chacun des deux strontiums.

La pratique de cette mesure est extrêmement laborieuse, car il faut faire le précipité oxalatique et les cendres, puis séparer $\mathrm{Ca} / \mathrm{Sr}$ par la différence de solubilité des deux nitrates, parfaire la séparation par colonne échangeuse d'ions, employer une autre colonne pour enlever tout l'yttrium préformé pour avoir au temps zéro du strontium pur, mesurer une première fois la radioactivité, séparer chimiquement l'yttrium provenant du strontium, après avoir attendu l'équilibre radioactif $\mathrm{Sr} 90 / \gamma 90$, ce qui demande 15 jours, et mesurer enfin la radioactivité due à l' $\gamma 90$. On a ainsi la radioactivité du $\mathrm{Sr} 90$ et par différence celle du $\mathrm{Sr} 89$.

D'autres méthodes par résines échangeuses d'ions ont été préconisées pour abréger ces manipulations. Actuellement, avec le vieillissement des retombées, il n'y a plus de Sr 89 qui a disparu par décroissance radioactive, ce qui simplifie considérablement la mesure.

Les résultats sont exprimés en pCi de Sr par litre de lait ou par pCi de Sr par gramme de Ca.

\section{Césium 137}

C'est un émetteur $\gamma$, on effectue la mesure au spectromètre. Le potassium sert d'étalon interne. Il faut partir de poudre de lait pour avoir une mesure convenable (voir fig. II). de $\mathrm{K}$.

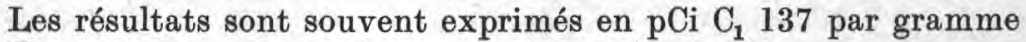

On voit que la mesure de la radiocontamination du lait est possible malgré la faible concentration de ces produits, mais ces techniques demandent du matériel coûteux et un personnel particulièrement bien qualifié.

\section{Moyens de défense contre les souillures radioactives du lait}

Contre ce danger de pollution du lait, quelles sont les mesures pour l'éviter ou le diminuer?

Elles sont extrêmement variées :

La meilleure et la plus sûre e'est l'arrêt des explosions nucléaires aériennes : l'accord russo-américain a été bénéfique, car depuis sa conclusion la pollution a diminué spectaculairement, il est maintenant à la limite de la sensibilité des appareils.

En cas de reprise des expériences nucléaires, ou d'accident sur un réacteur, si le taux de la radioactivité du lait atteignait un 
niveau dangereux, on ne serait pas désarmé, il faudrait agir sur le terrain, l'animal, le lait, enfin sur l'enfant et l'homme.

\section{Sur le terrain}

En chaulant les prés, on enrichit le sol en calcium et l'animal absorbe moins de strontium.

\section{Sur I'animal}

Il a été noté qu'une vache en élevage intensif avec, dans la ration, tous les suppléments requis, donnait un lait moins contaminé que celle en élevage extensif. L'équilibre de la ration joue un grand rôle. En nourrissant en partie les animaux avec du fourrage récolté en dehors des périodes de retombées, on diminue aussi la contamination. En fournissant à l'animal une ration d'iode stable qui va satisfaire les besoins de la thyroïde on évite la fixation de l'isotope radioactif.

\section{Sur le lait}

Il est possible, à partir du lait frais de faire du lait en poudre, de le conserver 3 mois, ce qui correspond à 10 périodes pour l'iode 131 : après ce laps de temps, l'activité en I 131 devient négligeable.

On peut, au moyen d'échangeurs d'ions ou par dialyse électrochimique, enlever du lait tout le calcium et le strontium qu'il contient. Dans un deuxième temps, on ajoute le calcium stable à la dose désirée. Ces appareils existent industriellement et ne posent pas de graves problèmes techniques.

On comprend pourtant que ces deux méthodes ne sont à la portée que des peuples à niveau de vie élevé. L'OMS et la FAO ont senti le danger et ont demandé que ces techniques ne soient employées qu'en cas d'accident ou de guerre nucléaire, autrement un peuple riche pourrait pratiquer des essais nucléaires sans aucune précaution en prenant à sa charge l'épuration du lait de ses nationaux, laissant les peuples voisins sous-développés, consommer un lait pollué.

\section{Sur l'enfant}

Il faut lui donner par priorité un lait propre, c'est-à-dire provenant d'animaux nourris d'aliments non contaminés; à défaut les enfants pourront recevoir du lait en poudre dialysé et stocké pendant 3 mois.

En période de crise, on peut enrichir artificiellement la ration alimentaire avec du calcium et de l'iode stables pour créer une compétition avec les isotopes radioactifs. 


\section{Sur l'homme}

C'est par un contrôle rigoureux du lait mis en vente que l'on peut éviter des accidents. Ce contrôle effectué par échantillonnage est très délicat à établir. Il faut un contrôle général assez souple s'étendant sur tout le territoire et un, beaucoup plus serré, entourant les points chauds : polygone de tirs, usine atomique.

Une technique qui tend à se développer consiste à mesurer la radioactivité fixée par les thyroïdes des bovidés : c'est une méthode extrêmement sensible, car il y a concentration de l'iode répandu sur une grande surface dans un organe très réduit. Elle est employée par des organismes officiels pour le contrôle général de la radioactivité du lait et autour des sites nucléaires où les bovidés sont régulièrement testés pour déceler une fuite accidentelle d'iode 131 et par extension des autres contaminants : e'est une véritable sonnette d'alarme.

Une autre méthode biologique est la mesure de la radioactivité des limnées, des escargots et de divers autres mollusques qui concentrent de grandes quantités de radionuclides dans leur hépato-pancréas.

\section{Conclusion}

Notons d'abord que les atomiciens n'ont jamais caché les dangers de cette radioactivité indésirable et par tous les moyens ils se sont ingéniés à la mesurer. Les résultats sont publiés régulièrement par les organismes officiels des divers pays : le risque peut done être suivi régulièrement.

Quel est-il actuellement?

Rassurons les diététiciens : jusqu'ici le seuil dangereux n'a jamais été atteint du fait des retombées. Depuis l'arrêt des explosions atomiques dans l'atmosphère, des Russes et des Américains, le taux de la radioactivité est extrêmement bas, la légère augmentation due aux bombes chinoises, tirées à la latitude de Madrid, n'a pas modifié cet état de fait; quant aux bombes françaises très étudiées et relativement faibles, elles souillent très peu l'atmosphère. Elles ont été expérimentées dans l'hémisphère austral ou près de l'équateur et les retombées possibles n'intéressent pas l'Europe.

Actuellement, on peut dire que le danger n'existe pas, mais il est potentiel et peut apparaître à nouveau brutalement, soit lors d'expériences nucléaires nouvelles, soit par accident, soit par fait de guerre. C'est pourquoi une connaissance du mécanisme des souillures radioactives du lait avec une évaluation exacte du danger est utile. Une surveillance continue s'impose car le lait est l'aliment de base-des enfants et de nombreux adultes.

La FAO et l'OMS et les experts officiels sont d'accord : si le contrôle de la radioactivité d'aliments devait être réduit du fait 
de la diminution du taux de la radioactivité ambiante, celui du lait serait conservé, car c'est le contrôle le plus important et le lait est l'aliment le plus directement menacé par les radio-éléments artificiels répandus par l'homme à la surface de la terre.

\section{Résumé}

Contamination du lait par les radio-éléments artificiels

L'auteur a étudié l'origine et le mécanisme de la contamination du lait, les principaux éléments de contamination et leur métabolisme, les méthodes de mesure de la contamination et enfin les moyens de défense.

En conclusion, il apparaît que le danger, actuellement négligeable, peut devenir réel et que le contrôle du lait doit être poursuivi car c'est l'aliment le plus menacé.

\section{Summary}

About contamination of milk by artificial radio-elements.

The author has studied origin and process of contamination of milk, the principal élément of the contamination and their metabolism, the measuring methods of this contamination and, finally, how we can oppose to it.

It appears that the danger, now neglectable, can become real and that the milk analysis must be caried on, for the milk is the most threatened food in this matter.

\section{BIBLIOGRAPHIE}

ANZani (A.). Mesure de la radioactivité ambiante à Ispra, 1961, Eur., 223. I., 1963.

FAIRMAN (W. D.). A rapid and sensitive method for the determination of I 131 in milk by ion exchange on silver chloride. Analy. Chem., $1966,38,9,1171$.

Fujita (M.) et coll. Relationship between ingestion, excretion and accumulation of fall out Cs 137 in man on a long term scale. Health Physics, $1966,12,12,1649$.

Grorcelli (F. G.). Contamination de la thyroïde des enfants italiens par ingestion d'I 131 provenant des retombées (octobre 1961-février 1963) (en italien). Minerva Nucleare, 1965, 9, 5, 325.

GLASCOCK (R. F.). Systems for removing radionuelides from milk Radioisotopes and radiation in dairy science and technology. Seminar Vienne, $12-15$ juillet $1966, \mathrm{SM}, 75 / 31$.

Jeanmaire (L.) et Michon (G.). Recherche et dosage des isotopes radioactifs 89 et 90 du strontium dans le lait en poudre. Le Lait, 1959, 39, 369.

JoHNSON (W. C.). Determination of radiostrontium by solvent extraction. Analy. Chem., 38, 8, 954. 
KNopp (E.). Etude bibliographique sur les possibilités et les procédés de décontamination radioactive du lait (en allemand), 1965, Eur. 2507.

MichoN (G.). Contrôle de la pollution radioactive du lait par l'iode 131. Bull. Acad. Vet., 1963, no 6, 26, 287.

MichoN (G.) et Madelmont (Cl.). Les pollutions radioactives du lait par

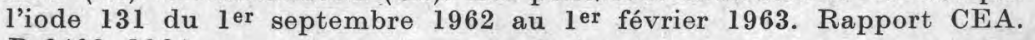
R 2409, 1964.

MorRe (J.) et Richou (L.). Hygiène alimentaire et radioactivité. A.V.H.A., éd., Paris, 1964.

Pellerin (P.), Rémy (M. L.) et coll. Premier bilan de 7 années de recherche sur les niveaux de contamination du milieu ambiant et de la chaîne alimentaire par les retombées radioactives sur le territoire français. $B u l l$. Inserm, 1967, 22, 2; SCPRI, 1967 (S), 115. 